\title{
The Effect of Probiotics on Symptoms, Gut Microbiota and Inflammatory Markers in Infantile Colic: A Systematic Review, Meta-Analysis and Meta- Regression of Randomized Controlled Trials
}

\author{
Karolina Skonieczna-Żydecka ${ }^{1}{ }^{\circledR}$, Katarzyna Janda ${ }^{1}$ (D), Mariusz Kaczmarczyk ${ }^{2}$, \\ Wojciech Marlicz ${ }^{3}$, Igor Łoniewski ${ }^{1}$ and Beata Łoniewska ${ }^{4, *}$ (D) \\ 1 Department of Human Nutrition and Metabolomics, Pomeranian Medical University in Szczecin, \\ 71-460 Szczecin, Poland; karzyd@pum.edu.pl (K.S.-Ż.); katarzyna.janda@pum.edu.pl (K.J.); \\ sanprobi@sanprobi.pl (I.Ł.) \\ 2 Department of Clinical and Molecular Biochemistry, Pomeranian Medical University in Szczecin, \\ 70-111 Szczecin, Poland; mariush@pum.edu.pl \\ 3 Department of Gastroenterology, Pomeranian Medical University in Szczecin, 71-252 Szczecin, Poland; \\ marlicz@hotmail.com \\ 4 Department of Neonatal Diseases, Pomeranian Medical University in Szczecin, 70-111 Szczecin, Poland \\ * Correspondence: beatal@pum.edu.pl; Tel.: +48(91)-466-1375
}

Received: 27 February 2020; Accepted: 30 March 2020; Published: 2 April 2020

\begin{abstract}
Immaturity in digestive-tract motor function and altered intestinal microbiome may play roles in pathogenesis of infantile colic. We assessed the impact of probiotic therapy on crying duration day, in newborns experiencing colic attacks. The PubMed, Embase, Cinnahl, Web of Science databases, and a clinical trials registry (ClinicalTrials.gov) were searched from inception until 12/02/2020. Random-effects meta-analyses were used to derive standardized mean differences/differences in means and risk ratios. We included 16 studies, which involved 1319 newborns aged up to 6 months. Lactobacillus reuteri strain DSM17938 was administered predominantly $(n=10)$. Probiotic intervention reduced the duration of crying (standardized mean difference $=-2.012,95 \%$ confidence interval: -2.763 to $-1.261, \mathrm{z}=-5.25, p<0.0001)$. The probability of at least a $50 \%$ reduction in crying duration was at least 1.98 times higher in the intervention group than in controls $(Z=4.80, p<0.0001)$. The effects of the intervention were not significantly affected by the risk of bias assessment, percentage of breastfed infants, and duration of the study. In 11 studies, data concerning gut microbiota composition and function and/or immunological markers were given. Probiotics significantly shortened the crying duration, but a causal relationship between the modulatory effect of probiotics on microbiota and the immune system has not been confirmed.
\end{abstract}

Keywords: infantile colic; probiotics; gut microbiota

\section{Introduction}

Infantile colic is a functional disorder of the gastrointestinal tract (point G4 in category G of the Rome IV Classification of Functional Gastrointestinal Disorders: Disorders of the Gut-Brain Interaction) [1-3]. Its apparent prevalence varies according to which diagnostic criteria are used and ranges from $2 \%$ to $73 \%$ [4]. The criteria for infantile colic were revised in the Rome IV classification and now, for clinical purposes, must include all of the following: (1) An infant who is less than 5 months of age when the symptoms start and finish; (2) Recurrent and prolonged periods of crying, fussing (see definition in Table S1), or irritability reported by caregivers that occur without obvious cause 
and which cannot be prevented or resolved by caregivers; (3) No evidence of failure to thrive, fever, or illness with clinical grounds (for details, see Table S1) [5].

Colicky ailments usually disappear in the first half-year of life, but before this happens, symptoms can bother parents, greatly resulting in repeated reporting to doctors [6-8]. The aetiology of intestinal colic in newborns is still poorly understood. It is presumed that the disorder results from a complex reaction between a newborn and the environment. Colic may also manifest as a result of immaturity of the structure and function of the gastrointestinal tract (GI) $[9,10]$. Both psychosociological factors, such as anxious parents, and gastrointestinal factors, such as allergy to cows' milk or gastroesophageal reflux (GER), also play pathophysiological roles [11-13].

One of the most recent and intensively analysed theories assumes that newborn colic is partly due to alterations of gut microbiota. There is a body of evidence that links a colicky phenotype [14] with skewed microbiota composition (e.g., lower diversity) [15-18], excess gas production, and altered GI motility. Moreover, colicky newborns $(\mathrm{CN})$ have been reported with alterations in bacterial abundance linked to gut inflammation and elevated levels of calprotectin and antimicrobial proteins released from intestinal neutrophils $[15-17,19,20]$. Of note, transplantation of $\mathrm{CN}^{\prime}$ 's faeces into mice resulted in visceral hypersensitivity to colorectal distention [21].

At least few interventions are known to alleviate excessive crying in colicky neonates, but with no evidence-based recommendations [22]. Historically, antispasmodic drugs, e.g., dicyclomine was found to counteract colicky behavior compared to placebo [23-25] but severe adverse effects, including coma [26], excluded the possibility to use the drugs in infants. As demonstrated in previous years $[4,27,28]$, anti-foaming agents (simethicone), enzyme-based (lactase), anticholinergicantimuscarinic-antispasmodic (cimetropium bromide, dicycloverine), opioids and antimuscarinic molecules (trimebutine), along with proton pump inhibitors (PPIs) showed some efficacy-as previously reported for simethicone $[29,30]$ - but confirmed potentially dangerous adverse effects for dicyclomine, cimetropium [31] and PPIs [32].

Several previous studies [19,33-36], carried out to find evidence of the efficacy of various probiotic strains in relieving the symptoms of infantile colic, have produced conflicting results. Overall, meta-analyses have reported that microbial probiotics, predominantly including Lactobacillus reuteri strains, have significantly shortened crying duration in CN [37-39]. However, none of the conducted studies analysed mechanisms potentially associated with probiotic intervention, including gut microbiome analysis, immunological parameters, and intestinal barrier function. Additionally, new original research data have recently been published, which were not included in previous systematic reviews and meta-analyses.

The main aim of the current study was to prepare an updated systematic review and meta-analysis to evidence the effectiveness of probiotics in the treatment of colic, in order to confirm the hypothesis that probiotics are superior to placebo and result in shorter crying duration in $\mathrm{CN}$. Additionally, in present metaanalysis, we have included studies looking for potential mechanisms of probiotics activity in $\mathrm{CN}$.

Moreover, meta-regression was also carried out regarding age and percentages of breastfed newborns and the quality of included studies, in order to explain contributing factors that may influence probiotic efficacy.

\section{Methods}

\subsection{Search Strategy and Inclusion Criteria}

Two independent authors (KSZ, KJ) searched PubMed/Embase/CINAHL/Web of Science databases and a clinical trials registry (www.clinicaltrials.gov) from inception until 12/02/2020 for randomized controlled trials (RCTs), which compared the effects of probiotics/synbiotics (hereafter referred to together as "probiotics") and placebos on reduction in crying duration in newborns manifesting colic attacks. The following search string was used in PubMed/Cinnahl/Web of Science: ("Probiotics"[MeSH] 
OR lactobacillus OR bifidobacterium OR saccharomyces OR streptococcus OR enterococcus OR bacillus) AND ("Colic"[MeSH] OR crying OR fussing OR irritable OR irritating). In Embase, the searched terms were: ('infant'/exp OR 'colic'/exp OR 'abdominal colic' OR 'colic' OR 'colicky pain' OR 'gastrointestinal colic') AND ('probiotic agent'/exp OR 'probiotic' OR 'probiotic agent' OR 'probiotics') AND ('placebo'/exp OR 'placebo') AND ('crying'/exp OR 'crying' OR 'weeping' OR 'fussiness'/exp). In the clinical trials registry, we used term "infantile colic" for condition and "probiotic" for other terms (https://clinicaltrials.gov/ct2/results?cond=infantile+colic\&term=probiotic\& cntry $=\&$ state $=\& c i t y=\&$ dist $=)$. The electronic search was followed by a manual screen of relevant reviews. Inclusion criteria were: (i) full-text randomized controlled trial conducted with full-term $\mathrm{CN}$; (ii) treatment with probiotic/synbiotic; (iii) randomization of probiotic vs. placebo; (iv) available meta-analyzable change score/endpoint data concerning crying duration (reduction in crying) during the day; and (v) English, German, or Polish text.

Data from studies containing more than two arms were collated separately for interventions. Additionally, if authors reported results for bottle-fed and breast-fed newborns separately, this representation was followed.

Exclusion criteria: (i) studies that randomized patients on more than one adjunctive intervention (e.g., probiotic plus proton pump inhibitor, probiotic incorporated into hydrolyzed casein formula); (ii) studies that did not conform to inclusion criteria.

\subsection{Data Abstraction and Outcomes}

We used the standard data extraction sheet according to our previous studies [40-42]. The following data from each study included were abstracted: study design, patient and treatment characteristics. For evaluation of the risk of bias (ROB) [43] we reported the number of low risk of bias assessments. Co-primary outcomes were crying duration per day and numbers of responders (defined as newborns who reduced daily crying by at least $50 \%$ compared to baseline) versus non-responders. These were assessed independently by at least two authors (K.S.-Z., K.J., I.Ł.), with one author (I.Ł.) acting as a dispute referee, in accordance with the Preferred Reporting Items for Systematic Reviews and Meta-Analyses (PRISMA). In the case of missing data, study authors were contacted via email. Data from figures was extracted by means of WebPlotDigitizer software (https://automeris.io/WebPlotDigitizer/).

\subsection{Data Synthesis and Statistical Analyses}

Using meta-analysis software (Comprehensive Meta-Analysis V3, Biostat, New Jersey, USA; www.meta-analysis.com), a random-effects [44] meta-analysis of outcomes, for which $\geq 3$ studies contributed data, was conducted. Heterogeneity by means using chi-square tests of homogeneity was evaluated. Analyses were two-tailed and alpha was equal to 0.05 .

For continuous outcomes, we analysed the pooled standardized mean difference (SMD) and difference in means (DM) in endpoint scores using observed cases (OC) data. For nominal outcomes the pooled risk ratio (RR), using OC data, was calculated. Meta-regression analyses with continuous (percentage of breastfed babies, study duration, number of low ROB assessments) covariates were conducted using random-effects models. The extent of asymmetries in funnel plots was detected using Egger's tests.

\section{Results}

\subsection{Search Results}

During the initial search, 1084 hits were found, of which 1036 were excluded at abstract level or as duplicates. The full-text review stage yielded 49 reviews. A total of 33 articles were excluded as they did not match inclusion criteria. We excluded all conference abstracts $(n=10)$. There were three studies $(n=3)$ conducted with premature newborns, which were excluded. Other reasons for exclusion were: studies aimed at preventing the incidence of colic $(n=4)$; co-intervention with other 
dietary approaches $(n=3)$; non-placebo comparators $(n=3)$; abstract with lack of full text availability $(n=1)$; commentary/editorials $(n=5)$, cows' milk based formula $(n=1)$, Iranian Language $(n=2)$, study protocol $(n=1)$. We were unable to find one study's full text $(n=1)$. In conclusion 16 studies were included in the final meta-analysis (see Figure 1, flow chart).

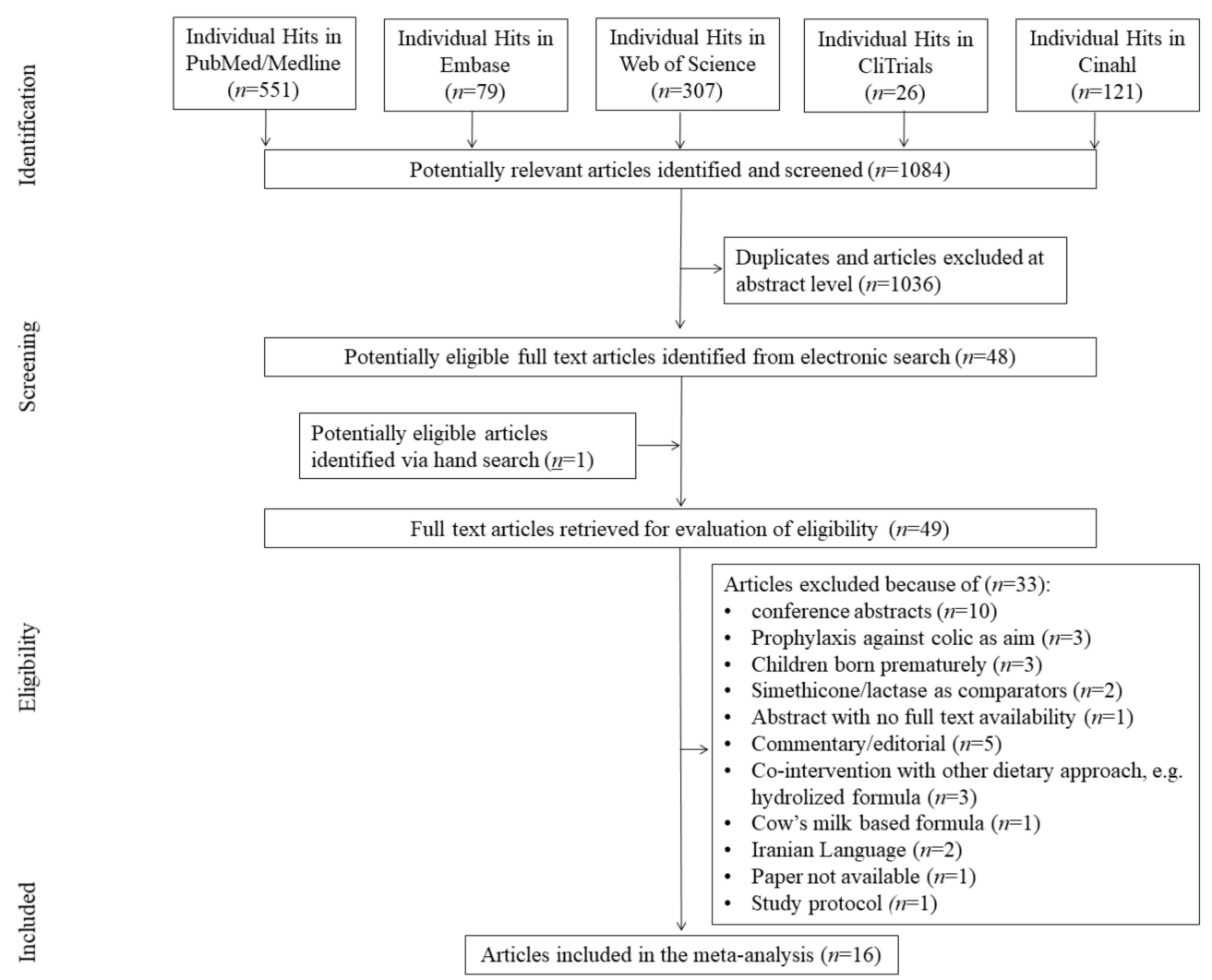

Figure 1. PRISMA flow diagram.

\subsection{Study, Patient, and Treatment Characteristics}

Ultimately 16 studies were included [19,33-35,45-56], the majority of which $(n=7)$ were conducted in Italy $[19,33,45,46,49,50,54]$. Intervention sponsorship was not specified for seven studies [19, $45,47,48,50,51,53]$ and two trials $[46,52]$ were financed by governmental institutions. There were 14 double-blinded studies [19,34,35,45-52,54-56], one single-blinded [53] and in one review, the blinding was not specified [33]. The duration of probiotic therapy was: median $=28$ days, interquartile range $(\mathrm{IQR})=8$ days, range $=14$ to 90 days. The primary probiotic strain utilized in the included studies was Lactobacillus reuteri Deutsche Sammlung von Mikroorganismen (German Collection of Microorganisms; DSM) strain $17938(n=9)$ [19,33-35,47,49,50,52,53,56]. Other probiotic strains were: Bifidobacterium breve BR03 (DSM16604) and B632 (DSM24706) $(n=1)$ [45], L. paracasei DSM24733, L. plantarum DSM24730, L. acidophilus DSM24735, L. delbrueckii subsp. bulgaricus DSM24734, B. longum DSM24736, B. breve DSM24732, B. longum ssp. infantis DSMv24737 and Streptococcus thermophilus DSM24731 $(n=1)$ [46], L. rhamnosus GG (American Type Culture Collection: ATCC53103), L. rhamnosus LC705 (DSM7061), B. longum ssp. infantis Bbi99 (DSM13692; given as B. breve in [48]), and Propionibacterium freudenreichii ssp. shermanii JS (DSM7067; $n=1$ ) [48]. A mix of L. rhamnosus 19070-2 and L. reuteri 12246 [55], and B. animalis subsp. lactis BB-12 ${ }^{\circledR}$, DSM 15954 were used in two studies, respectively [54]. In one study $(n=1)$, with a mixture of bacteria, there were no specific strain names given (L. casei, L. rhamnosus, Streptococcus thermophilus, B. breve, L. acidophilus, B. longum ssp. infantis, L. bulgaricus, and fructooligosaccharides) [51]. 
In total, 1319 newborns were randomized and 1215 analyzed, with a maximum of 172 newborns per study subjected to randomization and a mean \pm SD of $82.44 \pm 51.61$ per study with a similar mean number for analysis $(75.94 \pm 54.06)$. The numbers of males and females were similar (males $=52.73 \%$ ). In a majority of studies $(n=13)[19,33-35,46-51,53,55,56]$, infantile colic was diagnosed based on Wessel's or modified-Wessel's criteria. In one study, the Rome IV criteria were utilized [45], in one trial Rome III criteria [54] were used, and in one trial parental diaries were used for colic diagnoses [52]. As mentioned in the discussion, the inclusion criteria were only seemingly homogeneous (Table 1) and heterogeneity is discussed.

In 10 studies, newborns were breast-fed exclusively $[33,46-52,54,55]$ and in other studies the percentage of naturally-fed newborns ranged from $59.28 \%$ to $87.2 \%$, with the remaining newborns partly fed naturally and partly bottle-fed. Overall, probiotic intervention seemed to be well-tolerated as authors declared no adverse events regarding the intervention. In only one study [49], one newborn from a probiotic arm dropped out due to rhinitis, which was probably not related to the intervention. More study characteristics are shown in Table 1 and Table S2. 
Table 1. Study characteristics.

\begin{tabular}{|c|c|c|c|c|c|c|c|c|c|c|}
\hline \multirow{2}{*}{$\begin{array}{l}\text { Study } \\
\text { (Country) }\end{array}$} & \multicolumn{5}{|c|}{ Study Description } & \multicolumn{3}{|c|}{ Sample Description } & \multicolumn{2}{|c|}{ Intervention } \\
\hline & $\begin{array}{l}\text { Design, } \\
\text { Blinding }\end{array}$ & $\mathrm{ROB}^{\wedge}$ & $\begin{array}{l}\text { n Randomized/ } \\
\text { Analyzed }\end{array}$ & $\begin{array}{l}\text { Duration } \\
\text { (Days) }\end{array}$ & Study Focus & $\begin{array}{l}\text { Age (Mean } \\
\pm \text { SD, Days) }\end{array}$ & Male (\%) & $\begin{array}{l}\text { Breast-fed } \\
(\%)\end{array}$ & Probiotic Name & Probiotic Dose \\
\hline $\begin{array}{l}\text { Aloiso et al., } \\
2018 \text { [45] } \\
\text { (Italy) }\end{array}$ & DB & 4 & $158 / 155$ & 90 & $\begin{array}{l}\text { efficacy against functional } \\
\text { gastrointestinal disorders }\end{array}$ & $10.5 \pm 2.15$ & 52.26 & 83.87 & $\begin{array}{c}\text { B. breve BR03 (DSM } \\
\text { 16604) and B. breve B632 } \\
\text { (DSM 24706) }\end{array}$ & $\begin{array}{c}\text { drops } \\
\text { containing } 10^{8} \\
\text { CFU of each } \\
\text { strain }\end{array}$ \\
\hline $\begin{array}{l}\text { Baldassare et al., } \\
2018 \text { [46] (Italy) }\end{array}$ & $\mathrm{DB}$ & 7 & $62 / 53$ & 21 & efficacy in infantile colic & $38.75 \pm 1.72$ & 58.49 & 100 & $\begin{array}{c}\text { L. paracasei DSM } 24733 \text {, } \\
\text { L. plantarum DSM } 24730, \\
\text { L. acidophilus DSM } \\
\text { 24735, and L. delbrueckii } \\
\text { subsp. bulgaricus DSM } \\
\text { 24734), three strains of } \\
\text { bifidobacteria (B. } \\
\text { longum DSM 24736, B. } \\
\text { breve DSM 24732, and B. } \\
\text { longum ssp. infantis } \\
\text { DSM 24737), and one } \\
\text { strain of Streptococcus } \\
\text { thermophilus DSM } 24731\end{array}$ & $\begin{array}{c}5 \times 10^{9} \mathrm{CFU} / 10 \\
\text { drops }\end{array}$ \\
\hline $\begin{array}{c}\text { Chau et al., } 2015 \\
\text { [47] } \\
\text { (Canada) }\end{array}$ & DB & 7 & $55 / 52$ & 21 & efficacy in infantile colic & $39.73 \pm 0.32$ & 48.08 & 100 & L. reuteri DSM 17938 & $\begin{array}{l}10^{8} \mathrm{CFU} \text { in } 5 \\
\text { drops/day }\end{array}$ \\
\hline $\begin{array}{l}\text { Fatheree et al., } \\
2017 \text { [52] } \\
\text { (USA) }\end{array}$ & DB & 7 & $20 / 16$ & 42 & $\begin{array}{c}\text { efficacy against crying, } \\
\text { fussing, inflammatory, } \\
\text { immune, and microbiome } \\
\text { variables }\end{array}$ & $\begin{array}{l}\text { Probio: } 57 \\
\text { (39. } 72) ; \\
\text { PBO: } 40(34 . \\
\text { 51) }++ \\
\end{array}$ & 75 & 100 & L. reuteri DSM 17938 & $10^{8} /$ day \\
\hline $\begin{array}{l}\text { Gerasimov et al., } \\
2018 \text { [55] } \\
\text { (Ukraine) }\end{array}$ & DB & 5 & $172 / 168$ & 28 & $\begin{array}{l}\text { efficacy against crying, } \\
\text { fussing }\end{array}$ & $44.5 \pm 15$ & 50 & 100 & $\begin{array}{l}\text { L. rhamnosus } 19070-2, L \text {. } \\
\text { reuteri } 12246\end{array}$ & $\begin{array}{c}250 \times 10^{6} \mathrm{CFU}+ \\
3.33 \mathrm{mg} \\
\text { FOS+200IU } \\
\text { vitamin D3 }\end{array}$ \\
\hline
\end{tabular}


Table 1. Cont

\begin{tabular}{|c|c|c|c|c|c|c|c|c|c|c|}
\hline \multirow{2}{*}{$\begin{array}{l}\text { Study } \\
\text { (Country) }\end{array}$} & \multicolumn{5}{|c|}{ Study Description } & \multicolumn{3}{|c|}{ Sample Description } & \multicolumn{2}{|c|}{ Intervention } \\
\hline & $\begin{array}{r}\text { Design, } \\
\text { Blinding }\end{array}$ & $\mathrm{ROB}^{\wedge}$ & $\begin{array}{l}\text { n Randomized/ } \\
\text { Analyzed }\end{array}$ & $\begin{array}{l}\text { Duration } \\
\text { (Days) }\end{array}$ & Study Focus & $\begin{array}{c}\text { Age (Mean } \\
\pm \text { SD, Days) }\end{array}$ & Male (\%) & $\begin{array}{l}\text { Breast-fed } \\
(\%)\end{array}$ & Probiotic Name & Probiotic Dose \\
\hline $\begin{array}{l}\text { Kianifar et al., } \\
2014 \text { [51] } \\
\text { (Australia) }\end{array}$ & DB & 7 & $50 / 45$ & 30 & $\begin{array}{l}\text { reduction of newborns } \\
\text { crying over time }\end{array}$ & $42.17 \pm 17.38$ & 48.89 & 100 & $\begin{array}{l}\text { L. casei, L. rhamnosus, } \\
\text { Streptococcus } \\
\text { thermophilus, B. breve, } L \text {. } \\
\text { acidophilus, B. longum } \\
\text { ssp. infantis, L. } \\
\text { bulgaricus and FOS }\end{array}$ & $10^{9} \mathrm{CFU} /$ sachet \\
\hline $\begin{array}{l}\text { Mentula et al., } \\
2008 \text { [48] } \\
\text { (Finland) }\end{array}$ & DB & 0 & 18.wrz & 14 & $\begin{array}{l}\text { reduction of newborns } \\
\text { crying over time and } \\
\text { effect on gut microbiota }\end{array}$ & $21 \pm$ nd & 33.33 & 100 & $\begin{array}{l}\text { L. rhamnosus GG, L. } \\
\text { rhamnosus LC705, B. } \\
\text { longum ssp. infantis } \\
\text { Bbi99, and } \\
\text { Propionibacterium } \\
\text { freudenreichii ssp. } \\
\text { shermanii JS }\end{array}$ & $\begin{array}{c}\text { L. rhamnosus } \\
\text { GG:5 } \times 10^{9} \text { CFU; } \\
\text { L. rhamnosus } \\
\text { LC705: } 5 \times 10^{9} \\
\text { CFU; } \text { B. breve } \\
\text { Bbi99: } 2 \times 10^{8} \\
\text { CFU; P. } \\
\text { freudenreichii ssp. } \\
\text { shermanii JS: } 2 \times \\
10^{9} \text { CFU }\end{array}$ \\
\hline $\begin{array}{c}\text { Mi et al., } 2015 \\
\text { [53] } \\
\text { (China) }\end{array}$ & $\mathrm{SB}$ & 5 & $42 / 39$ & 28 & efficacy in infantile colic & $29.16 \pm 15.59$ & 56.41 & 87.18 & L. reuteri DSM 17938 & $10^{8} \mathrm{CFU}$ \\
\hline $\begin{array}{l}\text { Nation et al. } \\
2017 \text { [56] } \\
\text { (Australia) }\end{array}$ & DB & 5 & $167 / 167$ & 28 & $\begin{array}{l}\text { The relationship between } \\
\text { L. reuteri colonisation and } \\
\text { crying time, microbial } \\
\text { and inflammatory } \\
\text { parameters }\end{array}$ & $50.18 \pm 19.06$ & 50.89 & 59.28 & L. reuteri DSM 17938 & $\begin{array}{l}0.2 \times 10^{8} \\
\text { CFU/day }\end{array}$ \\
\hline $\begin{array}{l}\text { Nocerino et al., } \\
2020 \text { [54] (Italy) }\end{array}$ & DB & 7 & $80 / 78$ & 28 & efficacy in infantile colic & $32.95 \pm 5.15$ & 55.12 & 100 & $\begin{array}{c}\text { Bifidobacterium animalis } \\
\text { subsp. lactis BB-12 }{ }^{\circledR} \text {, } \\
\text { DSM } 15954\end{array}$ & $10^{9} \mathrm{CFU} /$ day \\
\hline $\begin{array}{l}\text { Savino et al. } \\
2010[49] \\
\text { (Italy) }\end{array}$ & DB & 6 & $50 / 46$ & 21 & $\begin{array}{l}\text { efficacy in infantile colic } \\
\text { and its relationship to the } \\
\text { gut microbiota }\end{array}$ & $\begin{array}{l}\text { PBO: } 28.5 \\
\text { (21) Probio: } \\
32.5(21) \dagger\end{array}$ & 63.04 & 100 & L. reuteri DSM 17938 & $10^{8} \mathrm{CFU}$ \\
\hline
\end{tabular}


Table 1. Cont

\begin{tabular}{|c|c|c|c|c|c|c|c|c|c|c|}
\hline \multirow{2}{*}{$\begin{array}{l}\text { Study } \\
\text { (Country) }\end{array}$} & \multicolumn{5}{|c|}{ Study Description } & \multicolumn{3}{|c|}{ Sample Description } & \multicolumn{2}{|c|}{ Intervention } \\
\hline & $\begin{array}{r}\text { Design, } \\
\text { Blinding }\end{array}$ & $\mathrm{ROB}^{\wedge}$ & $\begin{array}{l}\text { n Randomized/ } \\
\text { Analyzed }\end{array}$ & $\begin{array}{l}\text { Duration } \\
\text { (Days) }\end{array}$ & Study Focus & $\begin{array}{c}\text { Age (Mean } \\
\pm \text { SD, Days) }\end{array}$ & Male (\%) & $\begin{array}{l}\text { Breast-fed } \\
(\%)\end{array}$ & Probiotic Name & Probiotic Dose \\
\hline $\begin{array}{l}\text { Savino et al., } \\
2018 \text { [19] } \\
\text { (Italy) }\end{array}$ & DB & 5 & $87 / 60$ & 30 & $\begin{array}{c}\text { reduction of newborns } \\
\text { crying and modifying the } \\
\text { RORg/FOXP3 expression, } \\
\text { gut microbiota and faecal } \\
\text { calprotectin }\end{array}$ & $47.06 \pm 23.4$ & 43.33 & 83.33 & L. reuteri DSM 17938 & $\begin{array}{c}10^{8} \mathrm{CFU} / \text { drop; } 5 \\
\text { drops/day }\end{array}$ \\
\hline $\begin{array}{l}\text { Savino et al., } \\
\text { 2018a [50] (Italy) }\end{array}$ & DB & 5 & $59 / 30$ & 28 & $\begin{array}{c}\text { influence on Treg and } \\
\text { TLR expression (TLR } 2 \\
\text { and TLR4) }\end{array}$ & $26.4 \pm 12.36$ & 40 & 100 & L. reuteri DSM 17938 & $\begin{array}{l}0.2 \times 10^{8} \\
\text { CFU/drop; } 5 \\
\text { drops }\end{array}$ \\
\hline $\begin{array}{l}\text { Savino et al., } \\
2019 \text { [33] (Italy) }\end{array}$ & nd & 1 & $50 / 50$ & 28 & $\begin{array}{c}\text { influence on } \\
\text { CC-chemokine receptor } 7 \\
\text { (CCR7) and interleukin } 10 \\
\text { (IL-10) }\end{array}$ & $\begin{array}{l}\text { <50; PBO: } \\
\text { 28.5 (21) } \\
\text { Probio: } 32.5 \\
\quad(21) \dagger\end{array}$ & 58 & 100 & L. reuteri DSM 17938 & $\begin{array}{c}10^{8} \mathrm{CFU} / \text { drop; } 5 \\
\text { drops/day }\end{array}$ \\
\hline $\begin{array}{c}\text { Sung et al., } 2014 \\
{[35]} \\
\text { (Multicenter) }\end{array}$ & DB & 5 & $167 / 167$ & 28 & $\begin{array}{l}\text { reduction of newborns } \\
\text { crying and fussing }\end{array}$ & $50.18 \pm 19.06$ & 50.90 & 59.28 & L reuteri DSM 17938 & $\begin{array}{l}0.2 \times 10^{8} \\
\text { CFU/drop; } 5 \\
\text { drops/day }\end{array}$ \\
\hline $\begin{array}{l}\text { Szajewska et al., } \\
2013 \text { [34] } \\
\text { (Poland) }\end{array}$ & DB & 7 & $82 / 80$ & 21 & efficacy in infantile colic & $36.2 \pm 12.25$ & 60 & 86.25 & L. reuteri DSM 17938 & $\begin{array}{l}10^{8} \mathrm{CFU} \text { in } 5 \\
\text { drops }\end{array}$ \\
\hline
\end{tabular}

†—median (IQR); +†—median (Q1-Q3);^—number of low risk of bias (ROB) assessments; FOS—fructooligosaccharides; CFU—colony forming units; DB—double-blinded; Probio—probiotic; PBO—placebo; SB—single-blinded; nd—not determined, ${ }^{* * *}$ — partly same cohort as Sung et al., 2014 


\subsection{Risk of Bias (ROB)}

The mean number of low risk-of-bias assessments in all studies included in the meta-analysis was 5.19 (median = 5) $[19,34,35,46-56]$. There were six studies with the highest number, i.e., seven low ROB assessments $[34,46,47,51,52,54]$. Details of ROB evaluation are given in Table S3.

\subsection{Effects on Crying Duration and Response to Probiotic Intervention}

Using random-effects weights, the standardized mean difference for crying duration was -2.012 with a $95 \%$ confidence interval of -2.763 to -1.261 ( $z=-5.25, p<0.0001$; Figure 2). In case of difference in means, it was equal to -56.61 with a $95 \%$ confidence interval of -84.026 to -29.39 ( $z=-4.067$; $p<0.0001)$. No covariates were associated with study-level effects of probiotics on crying duration for both effect sizes, respectively (SMD - ROB: coefficient $=0.15$, standard error $(\mathrm{SE})=0.19, \mathrm{Z}=0.79$, $p=0.43$; breastfeeding percentage: coefficient $=-0.03, \mathrm{SE}=0.03, \mathrm{Z}=-1.20, p=0.23$; or duration of probiotic intervention: coefficient $=0.01, \mathrm{SE}=0.02, \mathrm{Z}=0.76, p=0.45 ; \mathrm{DM}$ - ROB: coefficient $=3.31$, standard error $(\mathrm{SE})=7.21, \mathrm{Z}=0.46, p=0.64$; breastfeeding percentage: coefficient $=-0.87, \mathrm{SE}=1.00$, $Z=-0.87, p=0.39$; or duration of probiotic intervention: coefficient $=0.65, S E=0.71, Z=2.05, p=$ $0.36)$.

Using random-effects analysis, the risk ratio for the eight studies was 1.98 with a $95 \%$ confidence interval of 1.5 to 2.62 . The probability of at least a $50 \%$ reduction in crying duration was at least 1.81 times higher in the intervention group than in the control group $(\mathrm{z}=4.804, p<0.0001$; Figure 3). A meta-regression using a random-effects model revealed no covariates regarding this study effect, respectively (ROB: coefficient $=-0.18, \mathrm{SE}=0.21, \mathrm{Z}=-0.84, p=0.40$; breastfeeding percentage: coefficient $=-0.04, \mathrm{SE}=0.02, \mathrm{Z}=-1.79, p=0.07$; or duration of probiotic intervention: coefficient $=$ $-0.03, \mathrm{SE}=0.02, \mathrm{Z}=-1.35, p=0.18$ ).

An Egger's test suggested a publication bias regarding the net effect of probiotics on crying duration (Egger's test - SMD: $p=0.0021$; DM: $p=0.007$; Figures 4 and 5) and there was no evidence of bias in the estimation of the response to intervention (Egger's test $p=0.50$; Figure S1). 


\section{Crying duration (min)}

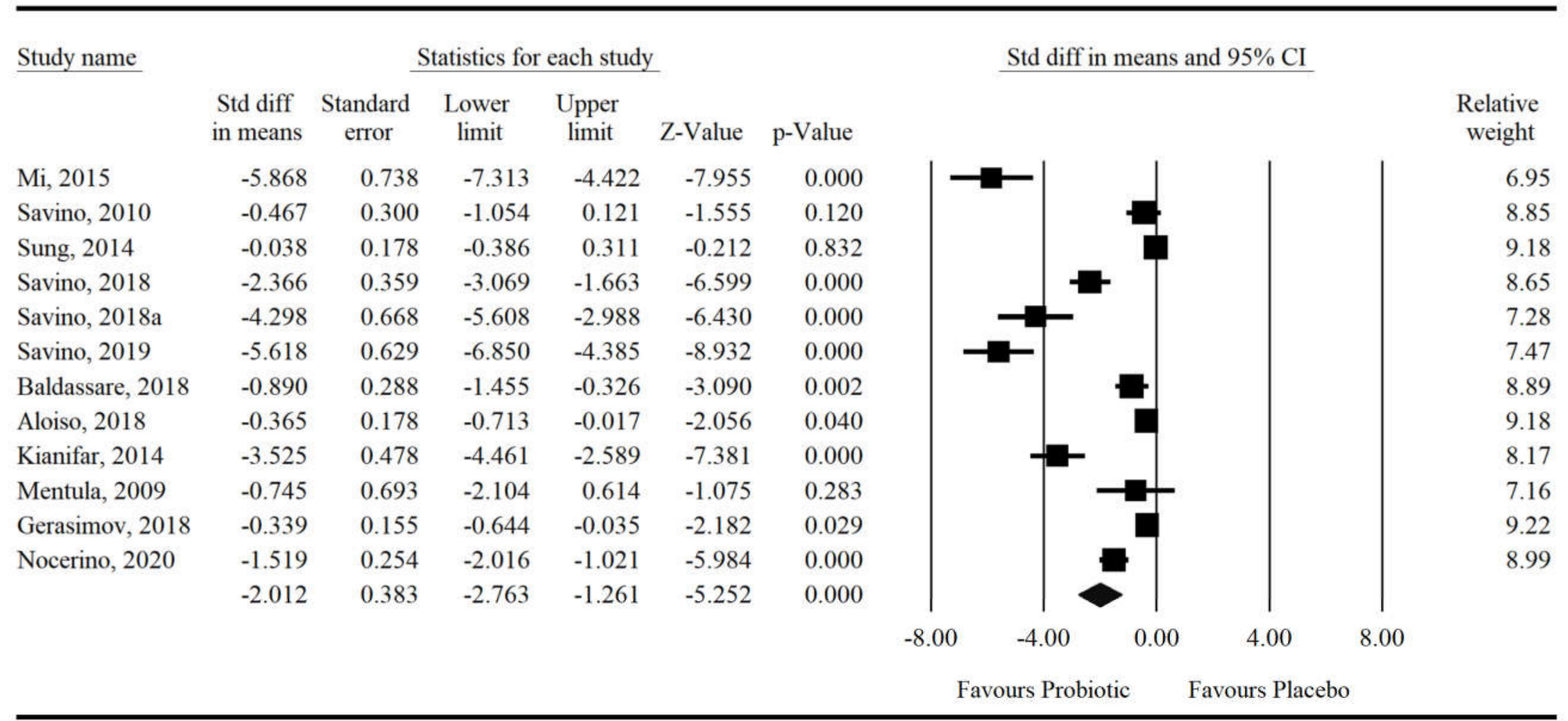

Figure 2. An effect size (random model), standardized mean difference, for crying duration in newborns taking probiotics vs. placebos (controls). $Q=227.3$, $\mathrm{df}(\mathrm{Q})=$ $11, p<0.001$, I-squared $=95.2$. 


\section{Responders}

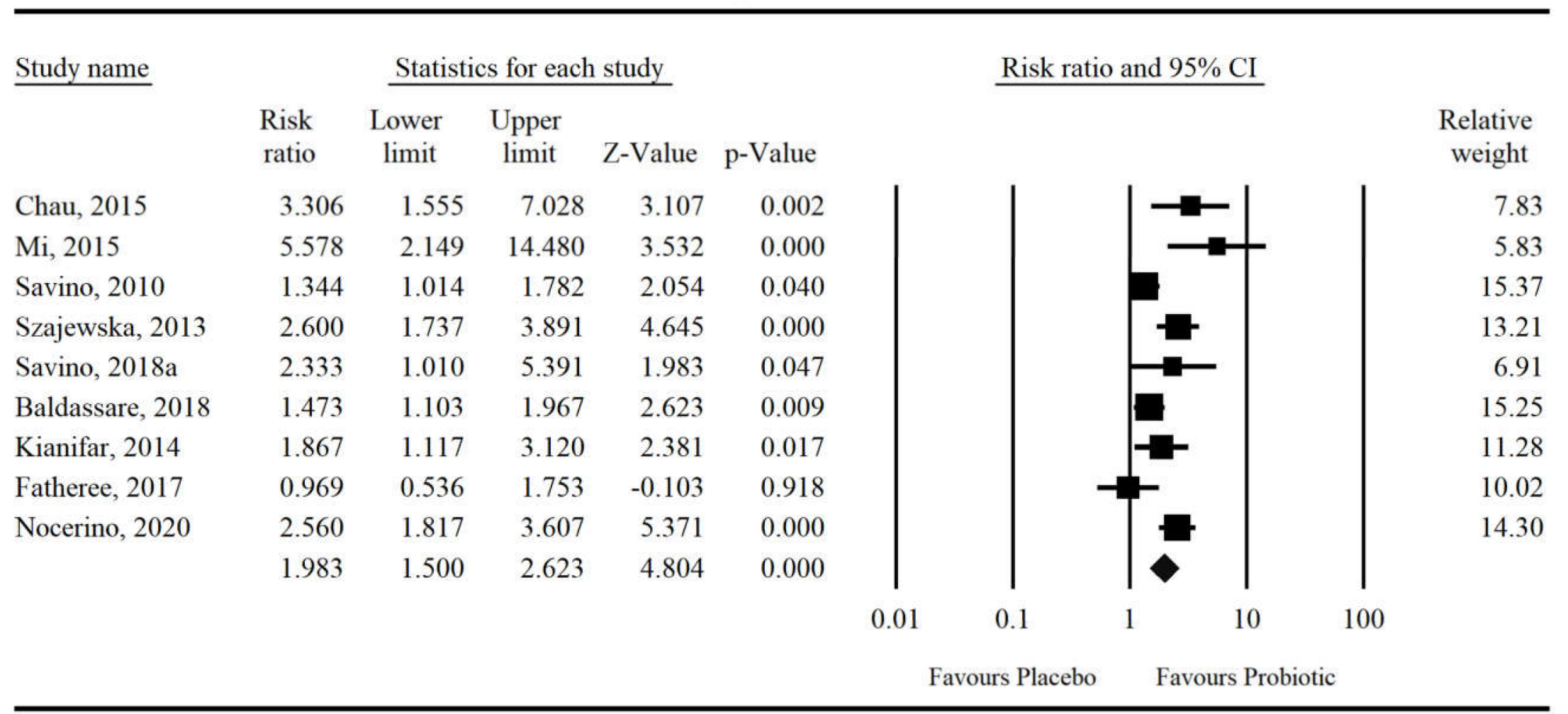

Figure 3. An effect size (random model), risk ratio, for the overall effects of probiotics with regard to a $50 \%$ reduction in crying duration. $Q=25.7, \mathrm{df}(\mathrm{Q})=8, p=0.001$,

I-squared $=68.9$. 
Funnel Plot of Standard Error by Std diff in means

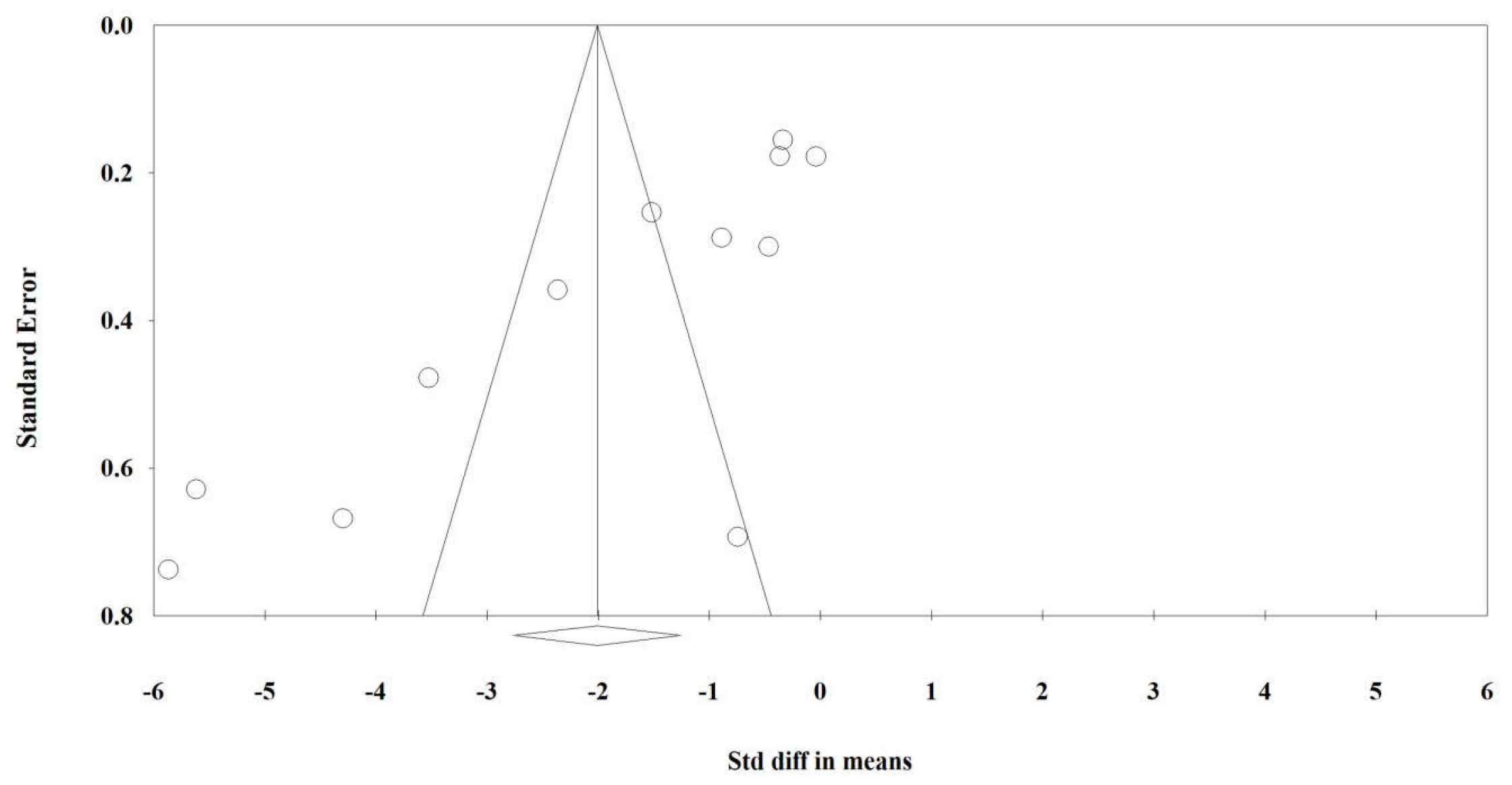

Figure 4. Funnel plot for crying time (SMD) in present meta-analysis. 


\section{Funnel Plot of Standard Error by Difference in means}

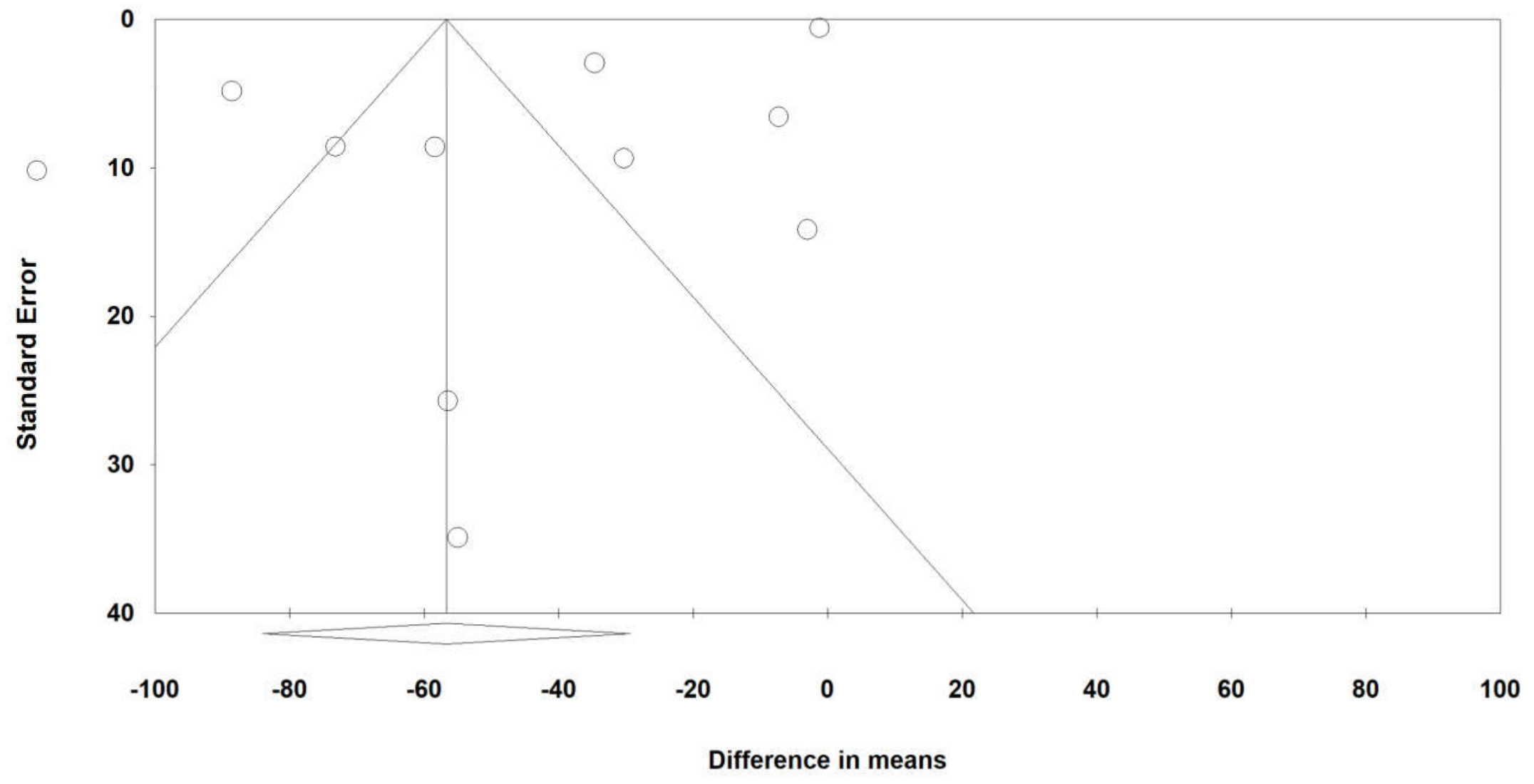

Figure 5. Funnel plot for crying time (DM) in present meta-analysis. 


\subsection{Microbiota and Immunological Parameters}

We found 11 trials $[19,33,35,45,46,48-50,52,54,56]$, in which data concerning gut microbiota composition and function and/or immunological markers were given. These were data on faecal microbiota composition $(n=9)$, metabolomic analyses $(n=4)$ as well as faecal (calprotectin $(n=4)$ ) and various blood immune $(n=5)$ markers. Table 2 presents major results on these parameters. Overall, aside from particular genera abundance $[19,33,35,45,46,48-50,52,54,56]$, basic microbiota metrics, as alpha diversity $[35,52,54]$, were reported. Only in three studies, microbiota by means of NGS technique was evaluated [35,52,54]. Other trials utilized a culture-dependent technique and qPCR. As one of the most important antimicrobial (and also a marker of gut inflammation and permeability $[57,58]$ is calprotectin, we extracted raw data on this protein levels were possible ( $n=5$ studies). We found that the level of calprotectin decreased by probiotic treatment $(n=2$ studies), but the data were presented in a not-metaanalyzable way (median, IQR) predominantly. These data are presented in Supplementary Table S4. We also analysed the association between clinical outcome, microbiota changes, and anti-inflammatory effects caused by probiotics administration (Supplementary Table S5). In three studies, clinical outcome was associated with microbial changes and in four studies with anti-inflammatory markers alterations. In three studies, changes in microbiota were observed, despite lack of clinical efficacy of probiotic treatment (in one study, crying time was even positively correlated with $L$. reuteri colonisation density). In two studies, statistical analysis of microbiota was not performed and in one study, the result of a metabolomic study was inconsistent (changes were also observed in the placebo group).

\section{Discussion}

This meta-analysis of 16 clinical trials and 1319 newborns exclusively investigated the impact of solely-probiotic interventions to reduce excessive crying in newborns manifesting symptoms of infantile colic, with no limitations on the age of study participants, and also gives a report on microbiota-related parameters affected by probiotics. Studies in which lactase, simethicone/herbal preparations or hydrolyzed formulas were utilized as probiotic comparators were not analyzed, as these interventions have their own evidence-based efficacy [59-64]. We also did not evaluate trials conducted with newborns who were not delivered at term, as premature newborns have been found to present immaturity in their digestive motility and interstitial Cajal cells [65] and are thus more prone to manifest behavioral perturbations [66]. By these means, we tried to diminish differences between populations of newborns in the articles studied which, as mentioned earlier, might have contributed to the uncertainty in probiotic efficacy found among previous reviews [67]. 
Table 2. Microbiological (compositional and functional) and inflammatory biomarkers affected by probiotics.

\begin{tabular}{|c|c|c|}
\hline Reference & Microbiota (Faeces)/Immunological Markers * & Method \\
\hline Aloisio et al., 2018 [45] & $\begin{array}{l}\text { - Whole cohort: } \uparrow \text { probiotic } B \text {. breve counts. Bottle fed newborns: } \downarrow \\
\text { potentially pathogenic } B \text {. fragilis group members. } \\
\text { - } \quad \text { Bifidobacterium spp. and } \downarrow \text { Clostridium spp over time with no } \\
\text { treatment effect, no differences in } E \text {. coli abundance over time. }\end{array}$ & qPCR \\
\hline Baldassare et al., 2018 [46] & $\begin{array}{l}\text { - No differences in total bacteria counts and Bifodobacterium spp. over } \\
\text { time and treatment. A statistical insignificant tendency toward } \\
\text { elevated Lactobacillus spp. with probiotic supplementation. } \\
\uparrow \text { faecal propylene glicol in probiotic group and } \uparrow \text { alanine, } \\
\text { 2-hydroxyisovalerate and 2-oxoisocaproate in placebo group. }\end{array}$ & qPCR/H-NMR \\
\hline Fatheree et al., 2017 [52] & $\begin{array}{l}\text { - } \quad \text { No differences in faecal alpha-diversity (Shannon, Chao1, or Simpson } \\
\text { diversity indices) over time and by treatment } \\
\text { - } \quad \downarrow \text { of dominant faecal gram (-) bacteria, i.e., Klebsiella, Proteus, and } \\
\text { Veillonella } \\
\text { - } \quad \downarrow \text { faecal calprotectin over time but not by the Tx } \\
\text { - } \quad \text { blood: } \downarrow \text { IL-2; } \downarrow \text { population of Tregs: } \mathrm{CD} 4^{+} \text {Foxp } 3^{+} \mathrm{CD}^{2} 5^{+} \text {within } \\
\mathrm{CD} 4^{+} \text {Foxp3 } 3^{+} \text {, and } \mathrm{CD}^{+} \mathrm{Foxp}^{+} \mathrm{HELIOS}^{+} \text {within } \mathrm{CD} 4^{+} \mathrm{Foxp}^{+} \\
\text {(thymus-derived) } \\
\text { with colic resolution in probiotic treated group (no statistical analysis } \\
\text { was done) }\end{array}$ & NGS/flow cytometry/ELISA \\
\hline Mentula et al., 2009 [48] & $\begin{array}{l}\text { - } \uparrow \text { of total counts of anaerobic bacteria, bifidobacteria, L. rhamnosus } \\
\text { GG, enterococci. } \\
\text { Faecal fermentation parameters (SCFA, CFA) were measured but no } \\
\text { statistical analysis was performed. }\end{array}$ & culture-dependent technique/GC \\
\hline *** Nation et al., 2017 [56] & $\begin{array}{l}\text { - } \quad \text { Crying time reduction regardless L. reuteri colonisation } \\
\text { - No differences in E. coli colonization rates or densities and microbial } \\
\text { diversity regarding L. reuteri colonization status. } \\
\text { - } \quad \text {. coli density negatively correlated with microbial diversity } \\
\text { - } \quad \text { L. reuteri concentration positively correlated with crying time } \\
\text { No difference in faecal calprotectin levels regarding probiotic } \\
\text { colonization status }\end{array}$ & qPCR/T-RFLP/ELISA \\
\hline
\end{tabular}


Table 2. Cont.

\begin{tabular}{|c|c|c|}
\hline Reference & Microbiota (Faeces)/ Immunological Markers & Method \\
\hline Nocerino et al., 2020 [54] & $\begin{array}{l}\text { - } \quad \text { No difference in microbiota composition and alpha-diversity index } \\
\text { by Tx } \\
\text { - } \quad \uparrow \text { Bifidobacterium spp. only in the responder infants treated with BB-12 } \\
\text { - } \quad \text { Bifidobacterium abundance was correlated with the reduction of } \\
\text { crying time } \\
\text { - } \quad \uparrow \text { Proteobacteria in the placebo group } \\
\text { - } \quad \uparrow \text { butyrate levels in responders } \\
\text { - } \quad \text { Blood: } \uparrow \text { HBD-2, LL-37, sIgA levels and } \downarrow \text { n faecal calprotectin level } \\
\text { in responders }\end{array}$ & $\begin{array}{l}\text { high-throughput sequencing of } 16 \mathrm{~S} \\
\text { rRNA, ELISA, indirect enzyme } \\
\text { immunoassays }\end{array}$ \\
\hline Savino et al., 2010 [57] & $\begin{array}{l}\text { - } \quad \uparrow \text { in Lactobacillus spp. (including probiotic L. reuteri) by Tx } \\
\text { - } \quad \downarrow \text { in faecal E. coli and ammonia }\end{array}$ & $\begin{array}{l}\text { culture-dependent techniques, } \\
\text { enzymatic colorimetric test }\end{array}$ \\
\hline Savino et al., 2018 [19] & $\begin{array}{ll}- & \uparrow \text { of Lactobacillus spp. by } \mathrm{T} \\
- & \downarrow \text { feacal calprotectin } \\
\text { - } & \text { Blood: } \uparrow \text { of FOXP3 concentration thus decreased RoRg/FOXP3 } \\
& \text { mRNA ratio }\end{array}$ & qPCR, ELISA \\
\hline Savino et al., 2018a [50] & - $\quad$ - Blood: $\uparrow$ mRNA expression of TREGs, FOXP3 & real time PCR/Qpcr \\
\hline Savino et al., 2019 [33] & $\begin{array}{ll} & -\uparrow \text { expression of } \text { CC-chemokine receptor } 7 \\
\end{array}$ & qPCR \\
\hline Sung et al., 2014 [58] & $\begin{array}{l}\text { - } \quad \text { No differences between in faecal microbial diversity, and E. coli load } \\
\text { by Tx } \\
\text { - } \quad \downarrow \text { feacal calprotectin in responders from probiotic and placebo group }\end{array}$ & $\begin{array}{l}\text { 16SrDNA amplification (T-RFLP) } \\
\text { ELISA, qPCR }\end{array}$ \\
\hline
\end{tabular}

IL-2 = interleukin 2; CD4+Foxp3+CD25+ =; CD4+Foxp3+HELIOS+ within CD4+Foxp3+ =; Helios-positive (thymus-derived) Tregs =; SCFA = short chain fatty acids; CFA = cellular fatty acids; HBD-2 = human $\beta$-defensin 2; LL-37 = cathelecidin; $\operatorname{sIgA}=$ secretory IgA; FOXP3 = forkhead box P3; ROR $\gamma=$ retinoid-related orphan receptor- $\gamma$; mRNA = messenger RNA; Th17 = T helper cell 17; Treg = regulatory T cell; qPCR = quantitative polymerase chain reaction; Tx - treatment; T-RFLP = terminal restriction fragment length polymorphism; NGS = next generation sequencing. rDNA = ribosomal DNA; ELISA = enzyme-linked immunosorbent assay; *-when not specified faecal biomarkers are listed; ***-partly same cohort as Sung et al., 2014 
The results of the present meta-analysis indicated that probiotics significantly reduced the duration of crying per day when compared to placebos-as evaluated in a random-effects model. We were also able to show that the percentages of treatment responders were higher in the probiotic group. Overall, our primary study results are similar to what has been found in previous meta-analyses. Schreck Bird et al. [39] analyzed the efficacy of two Lactobacillus reuteri strains versus placebo or simethicone in newborns delivered at term and predominantly breast-fed. These authors found that a $\sim 50 \%$ reduction in crying duration was $>2$ times more likely in newborns receiving probiotics. The authors, however, did not meta-analyze raw data concerning crying duration. Recently a similar study was published by Sung et al. [37], who evaluated L. reuteri DSM17938 as an agent that might relieve symptoms of infantile colic. The authors showed that after three weeks of intervention, the crying/fussing time decreased by almost half an hour. The incidence ratio to experienced relief was almost two times higher in the intervention group at different study points but significant in only breast-fed newborns. In the same year, a meta-analysis was conducted by Dryl and Szajewska [68] with seven RCTs and a cohort of 471 colicky participants, and found that crying reduction was significantly more probable in newborns taking probiotics and the number needed to treat was 5 (95\% CI: 4-8). On average, newborns receiving probiotics cried about 50 minutes shorter when compared to newborns taking placebos. Lactobacillus reuteri DSM17938 efficacy, however, seemed to be present exclusively in breast-fed newborns. The most recent study published in the Cochrane database [38] evaluated the efficacy of probiotics with colic prevention in newborns aged up to 1 month in comparison to placebo, but the authors were not able to prove an association. On the other hand, a subgroup meta-analysis of six RCTs and a cohort of 707 newborns with age of up to 1 month showed that the mean crying duration per day decreased by 32.6 minutes (44.3 minutes in studies utilizing Lactobacillus reuteri strains) in newborns receiving probiotics. Overall, the authors declared that no clear evidence of probiotic efficacy exists.

In our review, we found that probiotic effects were significant for both newborns exclusively breast-fed and newborns predominantly fed naturally; however, a meta-regression concerning the percentage of breastfed infants suggested that the efficacy of probiotics might be greater in infants fed with formulas, although the coefficient was extremely low (Coefficient $=-0.04 ; p=0.07$ ). In one study [45], included in our review, the authors reported that they had analyzed bottle- and breast-fed newborns separately but reported outcomes only in naturally-fed newborns. In another study [58], the crying duration was portrayed in such a way that meta-analysis adjustments were not possible concerning method of feeding. In the above-mentioned meta-analysis by Sung et al. [37], the authors found that the effects of probiotics were not significant in exclusively artificially-fed newborns, which cannot be confirmed by our review because we did not analyze exclusively bottle-fed newborns. As breastfeeding may potentially influence the efficacy of probiotics in IC, we can therefore make a presumption that the probiotic phenomenon might be associated with gut microbiota, which differ in naturally-fed newborns in comparison to artificially-fed newborns. Indeed, mother's milk contains its own microbiome [69,70] and also most importantly indigestible human milk oligosaccharides [71], which favourably affect the gut ecosystem [72]. Additionally, there are data that state that artificial feeding promotes the development of coliforms in $\mathrm{CN}$, and that this microbial print has not been demonstrated in breast-fed newborns with colic or newborns free of colic attacks [73]. On the other hand, if breast milk microbiota are affected by intrapartum antibiotics, in some cases, the beneficial effect of breast milk on the gut microbiome may be unfavourably altered [74]. Importantly, most authors did not report variables that may shape gut microbiota and intestinal permeability in newborns, e.g., mode of delivery, weight gain during pregnancy, antibiotic therapy during pregnancy and labor [75], which all may affect colicky behaviour. In addition, a body of evidence exists that links infantile colic with caffeine consumption [76] and smoking [77,78], as well as consumption of allergenic products [79]. Apart from in a few trials, we did not find much information regarding mother's health and lifestyle in the studies we included. In particular, in one study, the parents were advised to avoid cow's milk [49], while another excluded mothers taking antibiotics or those with allergy to cow's milk [50]. In a study 
by Sung et al. [35], the authors demonstrated that (by a small percentage difference) hypoallergenic formulas were more commonly used in the placebo group, as well as a dairy-free diet by mothers of breast-fed newborns. These might be factors that potentially biased the apparent overall effects of probiotics against prolonged colicky crying in breast-fed newborns.

Last but not least, we abstracted data related to influence of probiotics administration on gut microbiota and immunological markers. The most frequently studied variables were: (i) the effects of probiotic administration on the composition of the microbiota; (ii) colonization with probiotics, and (iii) the effect of reducing colonization with bacteria considered to be associated with colicky phenotype (e.g., E. coli [54]). Among immunological markers, mainly calprotectin and markers of Treg lymphocyte function were assessed. Based on the results obtained, no definite association can be found between the use of probiotics, microbiota changes, modulation of the immune system, and either presence or lack of clinical effects (Table 2 and Tables S4-S5). Of note, the results cannot be subjected to meta-analysis due to very diverse methods used to analyze the microbiota. Therefore, the results are difficult to compare. In addition, it is important to state that changes of microbiota are very dynamic in the first months and years of human life [80]. These observations are backed by studies assessing immunological markers such as calprotectin in this group of patients [81,82]. In addition, these markers are dependent on maternal-fetal factors, such as antibiotic therapy or BMI changes during pregnancy, mode of delivery, and feeding patterns [75]. For this reason, in order to fully assess the causal relationship between the microbiota and the function of the immune system with relation to symptoms of infantile colic, a multifactorial analysis should be performed, which was not performed in the works described in this systematic review. In only two studies, the correlation between microbiota changes and the effectiveness of probiotics was demonstrated $[54,56]$; however, the results were contradictory. In addition, the results of metabolomic studies did not contribute to elucidation of the mechanism of action of probiotics studied. Therefore, it cannot be determined whether the effect of probiotics in infantile colic is related to their effect on microbiota or the immune system. The relationship observed in some studies is rather based on association not causation. We conclude that mechanistic studies should be an important point in analysis of probiotics efficacy in $\mathrm{CN}$.

One study from the present meta-analysis diagnosed colic using Rome IV criteria [45] and the other Rome III criteria [54]. The rest utilised Wessel's or modified Wessel's criteria, and in one trial, a parental diary on crying duration was used. However, the reports on gut microbiota included in the above-mentioned papers all seem to be in line with the newest Rome IV criteria released in 2016 (Table S1). These criteria state that functional gastrointestinal disorders are the consequence of altered gut-brain interactions, an umbrella term for motility disorders, visceral hypersensitivity, immune dysfunction, microbiota alterations, and central nervous system alterations [2]. In a study based on Rome IV criteria [45], it was demonstrated that a probiotic strain, Lactobacillus reuteri DSM17938, may have had a positive impact on crying duration in $\mathrm{CN}$, underlining the hypothesis that gut-brain axis dysfunction may play a role in a colic phenotype. As has been documented that a major role of gut microbiota is to shape the structure and function of the gut and nervous system, we hope that the role of microbiota in this pathogenesis of $\mathrm{CN}$ will finally be elucidated, providing probiotics as a documented dietary option.

Several limitations of this meta-analysis require consideration. These include (i) a relatively small number of double-blinded studies comparing probiotic intervention to placebo; (ii) heterogeneous study inclusion criteria, particularly the age of newborns enrolled, and the type of strain and duration of probiotic intervention; (iii) the meta-regression analyses were only exploratory, as they were based on studies that may differ in newborn and treatment characteristics (delivery mode, antibiotic therapy as well as species/dosage/trial duration) from the overall sample in relevant ways.

In fact, all of these limitations may serve as confounding factors for probiotic efficacy; thus, to clearly show the efficacy of such microbial intervention, it is essential to conduct studies with restricted inclusion criteria, homogenous feeding, and probiotics to describe in great detail the impact of probiotics on colicky behavior. Additionally, these factors may have resulted in the asymmetrical 
funnel plot regarding crying duration. As elegantly reviewed by Sterne et al. [83], there are several possible explanations for such asymmetry, including reporting bias, poor methodological quality, true heterogeneity, as well as artifacts and chance. We carefully evaluated the risk of bias of each study and overall consider the quality of the included trials as high. We presume that intervention protocols differed significantly and this variable might remain as a so-called small study effect. Lastly, during meta-analysis, we did not use intent-to-treat data, which were not reported by authors but are preferred in clinical trials if the objective is pragmatic. We are also aware of potential bias during the review process, as we might have missed some studies not clearly aimed at reducing crying duration in $\mathrm{CN}$, but which might describe such outcomes.

\section{Conclusions}

Despite the limitations, this is a comprehensive meta-analysis evaluating solely-probiotic interventions compared to placebo, which showed that such dietary interventions are beneficial for $\mathrm{CN}$ and may counteract excessive crying. The mechanism of the action of probiotics in $\mathrm{CN}$ is still unknown. Based on the current analysis, we cannot assume that the action of probiotics is mediated either through modulation of microbiota or immune function. Relationships observed in some trials are rather based on associations and not causations. Therefore, further studies based on strict inclusion criteria are needed to clarify the role of probiotics in infantile colic and their impact on the gastrointestinal tract (e.g., intestinal barrier); and to compare the effectiveness of individual probiotic strains and doses, time of administration, and corresponding therapeutic effects.

Supplementary Materials: The following are available online at http://www.mdpi.com/2077-0383/9/4/999/s1, Table S1: Diagnostic criteria for newborn colic according to Rome IV, Rome II, and Wessel's criteria; Table S2: All-cause and adverse-effects-cause discontinuation within the trials; Table S3: Risk of bias assessment; Table S4: Faecal calprotectin levels $(\mu \mathrm{g} / \mathrm{g})$ by probiotic treatment; Table S5: Summary of the clinical outcome and changes in microbial composition and metabolites as well as anti-inflammatory effects associated with probiotics administration. Figure S1: Funnel plot for responding rate in present meta-analysis.

Author Contributions: Conceptualization, K.S.-Ż. and B.Ł.; Data curation, K.S.-Ż., K.J., M.K., W.M., and I.Ł.; Formal analysis, K.S.-Ż., M.K., W.M., I.Ł., and B.Ł.; Investigation, K.S.-Ż., K.J., W.M., and B.Ł.; Methodology, K.S.-Ż.; Project administration, B.Ł.; Resources, K.J., I.Ł., and B.Ł.; Software, K.S.-Ż. and M.K.; Supervision, I.Ł. and B.Ł.; Visualization, K.S.-Ż. and M.K.; Writing—original draft, K.S.-Ż.; Writing-review \& editing, K.J., M.K., W.M., I.Ł. and B.Ł. All authors have read and agreed to the published version of the manuscript.

Funding: This research received no external funding.

Conflicts of Interest: I.Ł. and W.M. are cofounders and shareholders at the Sanprobi company, a producer and distributor of probiotics. K.S.-Ż. and M.K. received honoraria from a probiotic company. However, it had no role in concept design, selection of articles, the decision to publish, or preparation of the manuscript. Other authors have nothing to disclose.

\section{References}

1. Wessel, M.A.; Cobb, J.C.; Jackson, E.B.; Harris, G.S.; Detwiler, A.C. Paroxysmal fussing in infancy, sometimes called colic. Pediatrics 1954, 14, 421-435. [PubMed]

2. Drossman, D.A.; Hasler, W.L. Rome IV-Functional GI Disorders: Disorders of Gut-Brain Interaction. Gastroenterology 2016, 150, 1257-1261. [CrossRef] [PubMed]

3. Benninga, M.A.; Faure, C.; Hyman, P.E.; St James Roberts, I.; Schechter, N.L.; Nurko, S. Childhood Functional Gastrointestinal Disorders: Neonate/Toddler. Gastroenterology 2016, 130, 1519-1526. [CrossRef]

4. Vandenplas, Y.; Abkari, A.; Bellaiche, M.; Benninga, M.; Chouraqui, J.P.; Çokura, F.; Harb, T.; Hegar, B.; Lifschitz, C.; Ludwig, T.; et al. Prevalence and Health Outcomes of Functional Gastrointestinal Symptoms in Infants From Birth to 12 Months of Age. J. Pediatr. Gastroenterol. Nutr. 2015, 61, 531-537. [CrossRef] [PubMed]

5. Palsson, O.S.; Whitehead, W.E.; van Tilburg, M.A.L.; Chang, L.; Chey, W.; Crowell, M.D.; Keefer, L.; Lembo, A.J.; Parkman, H.P.; Rao, S.S.; et al. Rome IV Diagnostic Questionnaires and Tables for Investigators and Clinicians. Gastroenterology 2016, 150, 1481-1491. [CrossRef] [PubMed] 
6. Rautava, P.; Helenius, H.; Lehtonen, L. Psychosocial predisposing factors for infantile colic. BMJ 1993, 307, 600-604. [CrossRef]

7. St James-Roberts, I.; Halil, T. Infant crying patterns in the first year: Normal community and clinical findings. J. Child Psychol. Psychiatry 1991, 32, 951-968. [CrossRef]

8. Zeevenhooven, J.; Koppen, I.J.N.; Benninga, M.A. The New Rome IV Criteria for Functional Gastrointestinal Disorders in Infants and Toddlers. Pediatr. Gastroenterol. Hepatol. Nutr. 2017, 20,1-13. [CrossRef]

9. Daelemans, S.; Peeters, L.; Hauser, B.; Vandenplas, Y. Recent advances in understanding and managing infantile colic. F1000Research 2018, 7. [CrossRef]

10. Sarasu, J.M.; Narang, M.; Shah, D. Infantile Colic: An Update. Indian Pediatr. 2018, 55, 979-987. [CrossRef]

11. Douglas, P.; Hill, P. Managing infants who cry excessively in the first few months of life. BMJ 2011, 343, d7772. [CrossRef] [PubMed]

12. Talge, N.M.; Neal, C.; Glover, V. Early Stress, Translational Research and Prevention Science Network: Fetal and Neonatal Experience on Child and Adolescent Mental Health Antenatal maternal stress and long-term effects on child neurodevelopment: How and why? J. Child Psychol. Psychiatry 2007, 48, 245-261. [CrossRef]

13. Van den Berg, M.P.; van der Ende, J.; Crijnen, A.A.M.; Jaddoe, V.W.V.; Moll, H.A.; Mackenbach, J.P.; Hofman, A.; Hengeveld, M.W.; Tiemeier, H.; Verhulst, F.C. Paternal depressive symptoms during pregnancy are related to excessive infant crying. Pediatrics 2009, 124, e96-e103. [CrossRef] [PubMed]

14. Gupta, S.K. Is colic a gastrointestinal disorder? Curr. Opin. Pediatr. 2002, 14, 588-592. [CrossRef] [PubMed]

15. De Weerth, C.; Fuentes, S.; Puylaert, P.; de Vos, W.M. Intestinal microbiota of infants with colic: Development and specific signatures. Pediatrics 2013, 131, e550-e558. [CrossRef] [PubMed]

16. Rhoads, J.M.; Fatheree, N.Y.; Norori, J.; Liu, Y.; Lucke, J.F.; Tyson, J.E.; Ferris, M.J. Altered fecal microflora and increased fecal calprotectin in infants with colic. J. Pediatr. 2009, 155, 823-828. [CrossRef]

17. Rhoads, J.M.; Collins, J.; Fatheree, N.Y.; Hashmi, S.S.; Taylor, C.M.; Luo, M.; Hoang, T.K.; Gleason, W.A.; Van Arsdall, M.R.; Navarro, F.; et al. Infant Colic Represents Gut Inflammation and Dysbiosis. J. Pediatr. 2018, 203, 55-61. [CrossRef]

18. Savino, F.; Cordisco, L.; Tarasco, V.; Calabrese, R.; Palumeri, E.; Matteuzzi, D. Molecular identification of coliform bacteria from colicky breastfed infants. Acta Paediatr. 2009, 98, 1582-1588. [CrossRef]

19. Savino, F.; Garro, M.; Montanari, P.; Galliano, I.; Bergallo, M. Crying Time and ROR $\gamma / F O X P 3$ Expression in Lactobacillus reuteri DSM17938-Treated Infants with Colic: A Randomized Trial. J. Pediatr. 2018, 192, 171-177. [CrossRef]

20. Savino, F.; Cresi, F.; Pautasso, S.; Palumeri, E.; Tullio, V.; Roana, J.; Silvestro, L.; Oggero, R. Intestinal microflora in breastfed colicky and non-colicky infants. Acta Paediatr. 2004, 93, 825-829. [CrossRef]

21. Eutamène, H.; Garcia-Rodenas, C.L.; Yvon, S.; d'Aldebert, E.; Foata, F.; Berger, B.; Sauser, J.; Theodorou, V.; Bergonzelli, G.; Mas, E. Luminal contents from the gut of colicky infants induce visceral hypersensitivity in mice. Neurogastroenterol. Motil. 2017, 29. [CrossRef] [PubMed]

22. Salvatore, S.; Barberi, S.; Borrelli, O.; Castellazzi, A.; Di Mauro, D.; Di Mauro, G.; Doria, M.; Francavilla, R.; Landi, M.; Martelli, A.; et al. Pharmacological interventions on early functional gastrointestinal disorders. Ital. J. Pediatr. 2016, 42, 68. [CrossRef] [PubMed]

23. Illingworth, R.S. Evening Colic in Infants: A Double-Blind Trial of Dicyclomine Hydrochloride. The Lancet 1959, 274, 1119-1120. [CrossRef]

24. Blomquist, H.K.; Mjörndal, T.; Tiger, G. Dicycloverin chloride solution-A remedy for severe infantile colic. Lakartidningen 1983, 80, 116-118. [PubMed]

25. Oggero, R.; Garbo, G.; Savino, F.; Mostert, M. Dietary modifications versus dicyclomine hydrochloride in the treatment of severe infantile colics. Acta Paediatr. 1994, 83, 222-225. [CrossRef]

26. Goldman, M.H. Dicycloverine for persistent crying in babies: Dicycloverine is contraindicated in infants. BMJ 2004, 328, 956. [CrossRef]

27. Shamir, R.; St James-Roberts, I.; Di Lorenzo, C.; Burns, A.J.; Thapar, N.; Indrio, F.; Riezzo, G.; Raimondi, F.; Di Mauro, A.; Francavilla, R.; et al. Infant crying, colic, and gastrointestinal discomfort in early childhood: A review of the evidence and most plausible mechanisms. J. Pediatr. Gastroenterol. Nutr. 2013, 57 (Suppl. 1), S1-S45. [CrossRef]

28. Bruyas-Bertholon, V.; Lachaux, A.; Dubois, J.-P.; Fourneret, P.; Letrilliart, L. Which treatments for infantile colics? La Presse Médicale 2012, 41, e404-e410. [CrossRef] 
29. Danielsson, B.; Hwang, C.P. Treatment of infantile colic with surface active substance (simethicone). Acta Paediatr. Scand. 1985, 74, 446-450. [CrossRef]

30. Metcalf, T.J.; Irons, T.G.; Sher, L.D.; Young, P.C. Simethicone in the treatment of infant colic: A randomized, placebo-controlled, multicenter trial. Pediatrics 1994, 94, 29-34.

31. Savino, F.; Brondello, C.; Cresi, F.; Oggero, R.; Silvestro, L. Cimetropium bromide in the treatment of crisis in infantile colic. J. Pediatr. Gastroenterol. Nutr. 2002, 34, 417-419. [CrossRef] [PubMed]

32. Gieruszczak-Białek, D.; Konarska, Z.; Skórka, A.; Vandenplas, Y.; Szajewska, H. No effect of proton pump inhibitors on crying and irritability in infants: Systematic review of randomized controlled trials. J. Pediatr. 2015, 166, 767-770. [CrossRef] [PubMed]

33. Savino, F.; Galliano, I.; Savino, A.; Daprà, V.; Montanari, P.; Calvi, C.; Bergallo, M. Lactobacillus reuteri DSM 17938 Probiotics May Increase CC-Chemokine Receptor 7 Expression in Infants Treated with for Colic. Front. Pediatr. 2019, 7, 292. [CrossRef] [PubMed]

34. Szajewska, H.; Gyrczuk, E.; Horvath, A. Lactobacillus reuteri DSM 17938 for the Management of Infantile Colic in Breastfed Infants: A Randomized, Double-Blind, Placebo-Controlled Trial. J. Pediatr. 2013, 162, 257-262. [CrossRef] [PubMed]

35. Sung, V.; Hiscock, H.; Tang, M.L.K.; Mensah, F.K.; Nation, M.L.; Satzke, C.; Heine, R.G.; Stock, A.; Barr, R.G.; Wake, M. Treating infant colic with the probiotic Lactobacillus reuteri: Double blind, placebo controlled randomised trial. BMJ 2014, 348, g2107. [CrossRef] [PubMed]

36. Indrio, F.; Mauro, A.D.; Riezzo, G.; Civardi, E.; Intini, C.; Corvaglia, L.; Ballardini, E.; Bisceglia, M.; Cinquetti, M.; Brazzoduro, E.; et al. Prophylactic Use of a Probiotic in the Prevention of Colic, Regurgitation, and Functional Constipation: A Randomized Clinical Trial. JAMA Pediatr. 2014, 168, 228-233. [CrossRef] [PubMed]

37. Sung, V.; D’Amico, F.; Cabana, M.D.; Chau, K.; Koren, G.; Savino, F.; Szajewska, H.; Deshpande, G.; Dupont, C.; Indrio, F.; et al. Lactobacillus reuteri to Treat Infant Colic: A Meta-analysis. Pediatrics 2018, 141, e20171811. [CrossRef]

38. Ong, T.G.; Gordon, M.; Banks, S.S.; Thomas, M.R.; Akobeng, A.K. Probiotics to prevent infantile colic. Cochrane Database Syst. Rev. 2019. [CrossRef]

39. Schreck Bird, A.; Gregory, P.J.; J alloh, M.A.; Risoldi Cochrane, Z.; Hein, D.J. Probiotics for the Treatment of Infantile Colic: A Systematic Review. J. Pharm. Pract. 2017, 30, 366-374. [CrossRef]

40. Skonieczna-Żydecka, K.; Kaczmarczyk, M.; Łoniewski, I.; Lara, L.F.; Koulaouzidis, A.; Misera, A.; Maciejewska, D.; Marlicz, W. A Systematic Review, Meta-Analysis, and Meta-Regression Evaluating the Efficacy and Mechanisms of Action of Probiotics and Synbiotics in the Prevention of Surgical Site Infections and Surgery-Related Complications. J. Clin. Med. 2018, 7, E556. [CrossRef]

41. Marlicz, W.; Skonieczna-Żydecka, K.; Yung, D.E.; Loniewski, I.; Koulaouzidis, A. Endoscopic findings and colonic perforation in microscopic colitis: A systematic review. Dig. Liver Dis. 2017, 49, 1073-1085. [CrossRef] [PubMed]

42. Skonieczna-Żydecka, K.; Łoniewski, I.; Misera, A.; Stachowska, E.; Maciejewska, D.; Marlicz, W.; Galling, B. Second-generation antipsychotics and metabolism alterations: A systematic review of the role of the gut microbiome. Psychopharmacology 2018, 236, 1491-1512.

43. Higgins, J.P.T.; Altman, D.G.; Gøtzsche, P.C.; Jüni, P.; Moher, D.; Oxman, A.D.; Savović, J.; Schulz, K.F.; Weeks, L.; Sterne, J.A.C. The Cochrane Collaboration's tool for assessing risk of bias in randomised trials. BMJ 2011, 343, d5928. [CrossRef] [PubMed]

44. DerSimonian, R.; Laird, N. Meta-analysis in clinical trials. Control Clin. Trials 1986, 7, 177-188. [CrossRef]

45. Aloisio, I.; Prodam, F.; Giglione, E.; Bozzi Cionci, N.; Solito, A.; Bellone, S.; Baffoni, L.; Mogna, L.; Pane, M.; Bona, G.; et al. Three-Month Feeding Integration with Bifidobacterium Strains Prevents Gastrointestinal Symptoms in Healthy Newborns. Front. Nutr. 2018, 5, 39. [CrossRef]

46. Baldassarre, M.E.; Di Mauro, A.; Tafuri, S.; Rizzo, V.; Gallone, M.S.; Mastromarino, P.; Capobianco, D.; Laghi, L.; Zhu, C.; Capozza, M.; et al. Effectiveness and Safety of a Probiotic-Mixture for the Treatment of Infantile Colic: A Double-Blind, Randomized, Placebo-Controlled Clinical Trial with Fecal Real-Time PCR and NMR-Based Metabolomics Analysis. Nutrients 2018, 10, 195. [CrossRef]

47. Chau, K.; Lau, E.; Greenberg, S.; Jacobson, S.; Yazdani-Brojeni, P.; Verma, N.; Koren, G. Probiotics for infantile colic: A randomized, double-blind, placebo-controlled trial investigating Lactobacillus reuteri DSM 17938. J. Pediatr. 2015, 166, 74-78. [CrossRef] 
48. Mentula, S.; Tuure, T.; Koskenala, R.; Korpela, R.; Könönen, E. Microbial composition and fecal fermentation end products from colicky infants-A probiotic supplementation pilot. Microb. Ecol. Health Dis. 2008, 20, $37-47$.

49. Savino, F.; Cordisco, L.; Tarasco, V.; Palumeri, E.; Calabrese, R.; Oggero, R.; Roos, S.; Matteuzzi, D. Lactobacillus reuteri DSM 17938 in Infantile Colic: A Randomized, Double-Blind, Placebo-Controlled Trial. Pediatrics 2010, 126, e526-e533. [CrossRef]

50. Savino, F.; Galliano, I.; Garro, M.; Savino, A.; Daprà, V.; Montanari, P.; Bergallo, M. Regulatory T cells and Toll-like receptor 2 and 4 mRNA expression in infants with colic treated with Lactobacillus reuteri DSM17938. Benef. Microbes 2018, 9, 917-925. [CrossRef]

51. Kianifar, H.; Ahanchian, H.; Grover, Z.; Jafari, S.; Noorbakhsh, Z.; Khakshour, A.; Sedaghat, M.; Kiani, M. Synbiotic in the management of infantile colic: A randomised controlled trial. J Paediatr. Child Health 2014, 50, 801-805. [CrossRef] [PubMed]

52. Fatheree, N.Y.; Liu, Y.; Taylor, C.M.; Hoang, T.K.; Cai, C.; Rahbar, M.H.; Hessabi, M.; Ferris, M.; McMurtry, V.; Wong, C.; et al. Lactobacillus reuteri for Infants with Colic: A Double-Blind, Placebo-Controlled, Randomized Clinical Trial. J. Pediatr. 2017, 191, 170-178. [CrossRef] [PubMed]

53. Mi, G.-L.; Zhao, L.; Qiao, D.-D.; Kang, W.-Q.; Tang, M.-Q.; Xu, J.-K. Effectiveness of Lactobacillus reuteri in infantile colic and colicky induced maternal depression: A prospective single blind randomized trial. Antonie Van Leeuwenhoek 2015, 107, 1547-1553. [CrossRef] [PubMed]

54. Nocerino, R.; De Filippis, F.; Cecere, G.; Marino, A.; Micillo, M.; Di Scala, C.; de Caro, C.; Calignano, A.; Bruno, C.; Paparo, L.; et al. The therapeutic efficacy of Bifidobacterium animalis subsp. lactis BB-12 ${ }^{\circledR}$ in infant colic: A randomised, double blind, placebo-controlled trial. Aliment. Pharmacol. Ther. 2020, 51, 110-120. [CrossRef] [PubMed]

55. Gerasimov, S.; Gantzel, J.; Dementieva, N.; Schevchenko, O.; Tsitsura, O.; Guta, N.; Bobyk, V.; Kaprus, V. Role of Lactobacillus rhamnosus (FloraActive ${ }^{\mathrm{TM}}$ ) 19070-2 and Lactobacillus reuteri (FloraActive ${ }^{\mathrm{TM}}$ ) 12246 in Infant Colic: A Randomized Dietary Study. Nutrients 2018, 10, 1975. [CrossRef]

56. Nation, M.L.; Dunne, E.M.; Joseph, S.J.; Mensah, F.K.; Sung, V.; Satzke, C.; Tang, M.L.K. Impact of Lactobacillus reuteri colonization on gut microbiota, inflammation, and crying time in infant colic. Sci. Rep. 2017, 7, 1-8. [CrossRef]

57. Peura, S.; Fall, T.; Almqvist, C.; Andolf, E.; Hedman, A.; Pershagen, G.; Helmersson-Karlqvist, J.; Larsson, A. Normal values for calprotectin in stool samples of infants from the population-based longitudinal born into life study. Scand. J. Clin. Lab. Invest. 2018, 78, 120-124. [CrossRef]

58. Albanna, E.A.; Ahmed, H.S.; Awad, H.A. Stool calprotectin in necrotizing enterocolitis. J. Clin. Neonatol. 2014, 3, 16.

59. Ahmed, M.; Billoo, A.G.; Iqbal, K.; Memon, A. Clinical Efficacy of Lactase Enzyme Supplement In Infant Colic: A Randomised Controlled Trial. J. Pak. Med. Assoc. 2018, 68, 1744-1747.

60. Biagioli, E.; Tarasco, V.; Lingua, C.; Moja, L.; Savino, F. Pain-relieving agents for infantile colic. Cochrane Database Syst. Rev. 2016. [CrossRef]

61. Kanabar, D.; Randhawa, M.; Clayton, P. Improvement of symptoms in infant colic following reduction of lactose load with lactase. J. Hum. Nutr. Diet. 2001, 14, 359-363. [CrossRef] [PubMed]

62. Martinelli, M.; Ummarino, D.; Giugliano, F.P.; Sciorio, E.; Tortora, C.; Bruzzese, D.; De Giovanni, D.; Rutigliano, I.; Valenti, S.; Romano, C.; et al. Efficacy of a standardized extract of Matricariae chamomilla L., Melissa officinalis L. and tyndallized Lactobacillus acidophilus (HA122) in infantile colic: An open randomized controlled trial. Neurogastroenterol. Motil. 2017, 29, e13145. [CrossRef] [PubMed]

63. Savino, F.; Pelle, E.; Palumeri, E.; Oggero, R.; Miniero, R. Lactobacillus reuteri (American Type Culture Collection Strain 55730) Versus Simethicone in the Treatment of Infantile Colic: A Prospective Randomized Study. Pediatrics 2007, 119, e124-e130. [CrossRef] [PubMed]

64. Stahlberg, M.R. Infantile colic: Occurrence and risk factors. Eur. J. Pediatr. 1984, 143, 108-111. [CrossRef]

65. Camilleri, M.; Park, S.-Y.; Scarpato, E.; Staiano, A. Exploring Hypotheses and Rationale for Causes of Infantile Colic. Neurogastroenterol. Motil. 2017, 29, e12943. [CrossRef]

66. Kusaka, R.; Ohgi, S.; Shigemori, K.; Fujimoto, T. Crying and Behavioral Characteristics in Premature Infants. J. Jpn. Phys. Ther. Assoc. 2008, 11, 15-21. [CrossRef]

67. Sung, V.; Collett, S.; de Gooyer, T.; Hiscock, H.; Tang, M.; Wake, M. Probiotics to prevent or treat excessive infant crying: Systematic review and meta-analysis. JAMA Pediatr. 2013, 167, 1150-1157. [CrossRef] 
68. Dryl, R.; Szajewska, H. Probiotics for management of infantile colic: A systematic review of randomized controlled trials. Arch. Med. Sci. 2018, 14, 1137-1143. [CrossRef]

69. Pannaraj, P.S.; Li, F.; Cerini, C.; Bender, J.M.; Yang, S.; Rollie, A.; Adisetiyo, H.; Zabih, S.; Lincez, P.J.; Bittinger, K.; et al. Association Between Breast Milk Bacterial Communities and Establishment and Development of the Infant Gut Microbiome. JAMA Pediatr. 2017, 171, 647-654. [CrossRef]

70. Moossavi, S.; Sepehri, S.; Robertson, B.; Bode, L.; Goruk, S.; Field, C.J.; Lix, L.M.; de Souza, R.J.; Becker, A.B.; Mandhane, P.J.; et al. Composition and Variation of the Human Milk Microbiota Are Influenced by Maternal and Early-Life Factors. Cell Host Microbe 2019, 25, 324-335. [CrossRef]

71. Dominguez-Bello, M.G.; Godoy-Vitorino, F.; Knight, R.; Blaser, M.J. Role of the microbiome in human development. Gut 2019, 68, 1108-1114. [CrossRef] [PubMed]

72. Thomas, D.W.; Greer, F.R. American Academy of Pediatrics Committee on Nutrition; American Academy of Pediatrics Section on Gastroenterology, Hepatology, and Nutrition Probiotics and prebiotics in pediatrics. Pediatrics 2010, 126, 1217-1231. [CrossRef] [PubMed]

73. Savino, F.; Ceratto, S.; Opramolla, A.; Locatelli, E.; Tarasco, V.; Amaretti, A. Coliforms and infant colic: Fish analysis of fecal samples of breastfed and formula fed infants. J. Pediatr. Gastroenterol. Nutr. 2013, 56, 472.

74. Hermansson, H.; Kumar, H.; Collado, M.C.; Salminen, S.; Isolauri, E.; Rautava, S. Breast Milk Microbiota Is Shaped by Mode of Delivery and Intrapartum Antibiotic Exposure. Front. Nutr. 2019, 6, 4. [CrossRef] [PubMed]

75. $\quad$ Łoniewska, B.; Węgrzyn, D.; Adamek, K.; Kaczmarczyk, M.; Skonieczna-Żydecka, K.; Adler, G.; Jankowska, A.; Uzar, I.; Kordek, A.; Celewicz, M.; et al. The Influence of Maternal-Foetal Parameters on Concentrations of Zonulin and Calprotectin in the Blood and Stool of Healthy Newborns during the First Seven Days of Life. An Observational Prospective Cohort Study. J. Clin. Med. 2019, 8, 473. [CrossRef]

76. McCreedy, A.; Bird, S.; Brown, L.J.; Shaw-Stewart, J.; Chen, Y.-F. Effects of maternal caffeine consumption on the breastfed child: A systematic review. Swiss Med. Wkly. 2018, 148, w14665. [CrossRef]

77. Shenassa, E.D.; Brown, M.-J. Maternal smoking and infantile gastrointestinal dysregulation: The case of colic. Pediatrics 2004, 114, e497-e505. [CrossRef]

78. Milidou, I.; Henriksen, T.B.; Jensen, M.S.; Olsen, J.; Søndergaard, C. Nicotine replacement therapy during pregnancy and infantile colic in the offspring. Pediatrics 2012, 129, e652-e658. [CrossRef]

79. Sjarif, D.R. Nutritional Intervention in Infantile Colic: Mini Review. IPCB 2017, 3, 266-272. [CrossRef]

80. Bäckhed, F.; Roswall, J.; Peng, Y.; Feng, Q.; Jia, H.; Kovatcheva-Datchary, P.; Li, Y.; Xia, Y.; Xie, H.; Zhong, H.; et al. Dynamics and Stabilization of the Human Gut Microbiome during the First Year of Life. Cell Host Microbe 2015, 17, 690-703. [CrossRef]

81. Lee, Y.M.; Min, C.-Y.; Choi, Y.J.; Jeong, S.J. Delivery and feeding mode affects fecal calprotectin levels in infants $<7$ months old. Early Hum. Dev. 2017, 108, 45-48. [CrossRef] [PubMed]

82. Li, F.; Ma, J.; Geng, S.; Wang, J.; Liu, J.; Zhang, J.; Sheng, X. Fecal Calprotectin Concentrations in Healthy Children Aged 1-18 Months. PLoS ONE 2015, 10, e0119574. [CrossRef] [PubMed]

83. Sterne, J.A.C.; Sutton, A.J.; Ioannidis, J.P.A.; Terrin, N.; Jones, D.R.; Lau, J.; Carpenter, J.; Rücker, G.; Harbord, R.M.; Schmid, C.H.; et al. Recommendations for examining and interpreting funnel plot asymmetry in meta-analyses of randomised controlled trials. BMJ 2011, 343, d4002. [CrossRef] [PubMed]

(C) 2020 by the authors. Licensee MDPI, Basel, Switzerland. This article is an open access article distributed under the terms and conditions of the Creative Commons Attribution (CC BY) license (http://creativecommons.org/licenses/by/4.0/). 